PROCEEDINGS OF THE AMERICAN MATHEMATICAL SOCIETY

Volume 124, Number 5, May 1996

\title{
DO ISOMORPHIC STRUCTURAL MATRIX RINGS HAVE ISOMORPHIC GRAPHS?
}

\author{
S. DǍSCĂLESCU AND L. VAN WYK
}

(Communicated by Lance W. Small)

\begin{abstract}
We first provide an example of a ring $R$ such that all possible $2 \times 2$ structural matrix rings over $R$ are isomorphic. However, we prove that the underlying graphs of any two isomorphic structural matrix rings over a semiprime Noetherian ring are isomorphic, i.e. the underlying Boolean matrix $B$ of a structural matrix ring $\mathbb{M}(B, R)$ over a semiprime Noetherian ring $R$ can be recovered, contrary to the fact that in general $R$ cannot be recovered.
\end{abstract}

\section{INTRODUCTION AND PRELIMINARIES}

Various authors have studied the relationship between two rings $R$ and $S$ for which the full matrix rings $\mathbb{M}_{n}(R)$ and $\mathbb{M}_{n}(S)$ are isomorphic. For example, Chatters [1] showed that one is very far from recovering the ring $R$ from $\mathbb{M}_{n}(R)$, even in the prime Noetherian case: he provided an uncountable family of pairwise nonisomorphic such rings with isomorphic full $2 \times 2$ matrix rings.

Certain subrings of $\mathbb{M}_{n}(R)$, for example tiled matrix rings, have also recently received considerable attention (see [4]). A particular class of tiled matrix rings are the structural matrix rings, having the tiles 0 or $R$. Instances of such rings have been intensively studied. For example, the automorphisms of structural matrix rings over certain rings were described in [2] and [3], and a left Artinian CI-prime ring was characterised as a complete blocked triangular matrix ring over a division ring in [5].

The properties of a structural matrix ring $\mathbb{M}(B, R)$, being a generalisation of both a full matrix ring and an upper triangular matrix ring, are influenced by the underlying Boolean matrix $B$ (or, equivalently, the underlying graph) and the underlying ring $R$. We noted in the first paragraph that it is not in general possible to recover the ring $R$ from $\mathbb{M}_{n}(R)$. However, it might be possible to recover the Boolean matrix $B$ from the structural matrix ring $\mathbb{M}(B, R)$. There are of course trivial examples of isomorphic structural matrix rings over a ring $R$ having Boolean matrices of different orders, viz. consider any ring $R$ for which $R \cong R \times R$. Moreover, we provide an example of a ring $R$ for which all the $2 \times 2$ structural matrix rings (i.e. the Boolean matrices have the same order) are isomorphic. However, for certain rings this phenomenon cannot occur. The main result of this paper states

Received by the editors December 7, 1993 and, in revised form, November 3, 1994.

1991 Mathematics Subject Classification. Primary 16S50, 16P40, 16N60.

Key words and phrases. Structural matrix ring, semiprime Noetherian ring, Boolean matrix, graph. 
that any two isomorphic structural matrix rings over a semiprime Noetherian ring have conjugated Boolean matrices, i.e. one of them can be obtained from the other by a permutation of the rows and columns, and this is equivalent to saying that their associated graphs are isomorphic (Theorem 2.4). We conclude that the same result holds over any commutative Noetherian ring (Corollary 2.5).

Throughout the paper every ring will be associative with identity, and the identity is assumed to be inherited by all subrings. We denote the prime radical of a ring $R$ by $\mathcal{P}(R)$ and the uniform dimension of $R$ as a left $R$-module by $\mathrm{u} \operatorname{dim} R$. By a Noetherian ring we mean a left noetherian ring, and by a Boolean matrix we mean a reflexive and transitive $n \times n$ Boolean matrix $B=\left[b_{i, j}\right]$, i.e. $b_{i, j} \in\{0,1\}$, $b_{i, i}=1$ for every $i=1, \ldots, n$, and if $b_{i, j}=b_{j, k}=1$, then $b_{i, k}=1$. The order $n$ of $B$ will be denoted by $\operatorname{order}(B)$. We call $B$ blocked triangular if it is of the form

$$
\left[\begin{array}{cccc}
X_{1,1} & X_{1,2} & \ldots & X_{1, t} \\
0 & X_{2,2} & \ldots & X_{2, t} \\
\vdots & \ddots & \ddots & \vdots \\
0 & \ldots & 0 & X_{t, t}
\end{array}\right],
$$

where for every $i \leq j, X_{i, j}$ is an $n_{i} \times n_{j}$ (Boolean) matrix with all its entries equal, and $n_{1}+\cdots+n_{t}=n$. Since we assume $B$ to be reflexive, every entry of $X_{i, i}$ is 1 , $i=1, \ldots, t$. We call $X_{i, j}$ the $(i, j)$ th block and $X_{i, k}$ the $i$ th diagonal block of $B$. If every entry of $X_{i, j}$ is 1 for all $i \leq j$, then $B$ is called complete blocked triangular (see $[5])$.

If $B=\left[b_{i, j}\right]$ is a Boolean matrix and $\sigma \in S_{n}$ a permutation (of the set $\{1, \ldots, n\}$ ), then we denote by $\sigma(B)$ the Boolean matrix with $b_{i, j}$ in position $(\sigma(i), \sigma(j)), i, j=$ $1, \ldots, n$. It is easy to see that for any Boolean matrix $B$ there is a permutation $\sigma \in S_{n}$ such that $\sigma(B)$ is blocked triangular. If $\varphi\left(\left[r_{i j}\right]\right),\left[r_{i j}\right] \in \mathbb{M}(B, R)$, is the matrix with $r_{i j}$ in position $(\sigma(i), \sigma(j))$, then $\varphi: \mathbb{M}(B, R) \rightarrow \mathbb{M}(\sigma(B), R)$ is a ring isomorphism. Here, $\mathbb{M}(B, R)$ is the structural matrix ring (associated with $B$ and $R$ ), i.e. the subring of $\mathbb{M}_{n}(R)$ comprising all matrices having 0 in the position $(k, l)$ whenever $b_{k, l}=0$.

With any Boolean matrix $B$ we associate a directed graph $\Gamma(B)$ with vertices $1, \ldots, n$ and an arc from $i$ to $j(1 \leq i, j \leq n)$ whenever $b_{i, j}=1$. We denote the undirected graph associated with $\Gamma(B)$ by $\Gamma^{\prime}(B)$. A connected component of $B$ is the (square) submatrix of $B$ determined by a connected component of $\Gamma^{\prime}(B)$, and $B$ is called connected if $\Gamma^{\prime}(B)$ is connected. Henceforth graph will mean directed graph. If $B_{1}$ and $B_{2}$ are Boolean matrices, then clearly the graphs $\Gamma\left(B_{1}\right)$ and $\Gamma\left(B_{2}\right)$ are isomorphic if and only if there is a permutatin $\sigma \in S_{n}$ such that $B_{2}=\sigma\left(B_{1}\right)$.

We start by providing an example of a ring $R$ such that all possible $2 \times 2$ structural matrix rings over $R$ are isomorphic.

Example 1.1. We construct a ring $R$ such that

$$
\left[\begin{array}{cc}
R & 0 \\
0 & R
\end{array}\right] \cong\left[\begin{array}{cc}
R & R \\
0 & R
\end{array}\right] \cong\left[\begin{array}{ll}
R & 0 \\
R & R
\end{array}\right] \cong\left[\begin{array}{ll}
R & R \\
R & R
\end{array}\right] \cong R
$$

To this end, let $I:=\{p: p \in[0,1)$ and $p$ has a finite representation in base 2$\}$. (We mean that if $p=p_{1} / 2+\cdots+p_{m} / 2^{m}$ for some $m$, with $p_{1}, \ldots, p_{m} \in\{0,1\}$, then the representation of $p$ is $0 . p_{1} \cdots p_{m}$.) Let $\mathbb{M}_{I}(K)$ be the ring of column-finite $I \times I$ matrices over $K, K$ a ring, i.e. every column of a matrix in $\mathbb{M}_{I}(K)$ has at most a finite number of nonzero entries. Let $S$ be the subring of $\mathbb{M}_{I}(K)$ comprising the 
matrices with 0 in position $(p, q)$ whenever $p_{i}=1$ and $q_{i}=0$ for some $i \geq 1$ (with $\left.q=0 . q_{1} \cdots q_{n}\right)$. Let $V\left(=\left[v_{p, q}\right]\right), W, X, Y \in \mathbb{M}_{I}(K)$, and define

$$
\varphi:\left[\begin{array}{ll}
\mathbb{M}_{I}(K) & \mathbb{M}_{I}(K) \\
\mathbb{M}_{I}(K) & \mathbb{M}_{I}(K)
\end{array}\right] \rightarrow \mathbb{M}_{I}(K)
$$

by setting

$$
\varphi\left(\left[\begin{array}{ll}
V & W \\
X & Y
\end{array}\right]\right)=: Z
$$

where $Z$ is defined by

$$
\begin{aligned}
& z_{0.0 p_{1} \cdots p_{m}, 0.0 q_{1} \cdots q_{n}}=v_{0 . p_{1} \cdots p_{m}, 0 . q_{1} \cdots q_{n}}, \\
& z_{0.0 p_{1} \cdots p_{m}, 0.1 q_{1} \cdots q_{n}}=w_{0 . p_{1} \cdots p_{m}, 0 . q_{1} \cdots q_{n}}, \\
& z_{0.1 p_{1} \cdots p_{m}, 0.0 q_{1} \cdots q_{n}}=x_{0 . p_{1} \cdots p_{m}, 0 . q_{1} \cdots q_{n}}, \\
& z_{0.1 p_{1} \cdots p_{m}, 0.1 q_{1} \cdots q_{n}}=y_{0 . p_{1} \cdots p_{m}, 0 . q_{1} \cdots q_{n}} .
\end{aligned}
$$

Then direct verification shows that $\varphi$ is a ring isomorphism, and the restriction of $\varphi$ to $\left[\begin{array}{cc}S & S \\ 0 & S\end{array}\right]$ has the image $S$. Hence we have a ring $S$ such that

$$
S \cong\left[\begin{array}{cc}
S & S \\
0 & S
\end{array}\right] \text {. }
$$

Let $J$ be an infinite set and set $T:=S^{J}$, the direct product of $J$ copies of $S$. Then clearly by (2)

$$
T \times T \cong T \cong\left[\begin{array}{cc}
S & S \\
0 & S
\end{array}\right]^{J} \cong\left[\begin{array}{cc}
S^{J} & S^{J} \\
0 & S^{J}
\end{array}\right]=\left[\begin{array}{cc}
T & T \\
0 & T
\end{array}\right] .
$$

If $R:=\mathbb{M}_{J}(T)$, then clearly by (3)

$$
R \cong \mathbb{M}_{J}(T \times T) \cong \mathbb{M}_{J}(T) \times \mathbb{M}_{J}(T)=R \times R .
$$

Furthermore, by (3)

$$
R \cong \mathbb{M}_{J}\left(\left[\begin{array}{ll}
T & T \\
0 & T
\end{array}\right]\right) \cong\left[\begin{array}{cc}
\mathbb{M}_{J}(T) & \mathbb{M}_{J}(T) \\
0 & \mathbb{M}_{J}(T)
\end{array}\right]=\left[\begin{array}{cc}
R & R \\
0 & R
\end{array}\right] .
$$

Moreover, since $J$ is infinite, we have

$$
\mathbb{M}_{J}(T) \cong\left[\begin{array}{ll}
\mathbb{M}_{J}(T) & \mathbb{M}_{J}(T) \\
\mathbb{M}_{J}(T) & \mathbb{M}_{J}(T)
\end{array}\right], \quad \text { i.e. } R \cong\left[\begin{array}{ll}
R & R \\
R & R
\end{array}\right],
$$

and so (1) follows from (4), (5) and (6).

\section{StruCtural Matrix Rings OVER SEMIPRIme Noetherian RINGS}

We first take care of the orders of the diagonal blocks of the underlying Boolean matrices of two isomorphic structural matrix rings over a Noetherian ring.

Lemma 2.1. Let $B_{1}$ and $B_{2}$ be blocked triangular Boolean matrices, and let $R$ be a Noetherian ring such that $\mathbb{M}\left(B_{1}, R\right) \cong \mathbb{M}\left(B_{2}, R\right)$. If $n_{1}, \ldots, n_{t}$ and $m_{1}, \ldots, m_{s}$ are the orders of the diagonal blocks of $B_{1}$ and $B_{2}$ respectively, then $t=s$ and there is a permutation $\sigma \in S_{t}$ such that $m_{i}=n_{\sigma(i)}, i=1, \ldots, t$. 
Proof. By [7, Theorem 2.7] $\mathcal{P}\left(\mathbb{M}\left(B_{i}, R\right)\right)$ comprises the matrices in $\mathbb{M}\left(B_{i}, R\right)$ having elements of $\mathcal{P}(R)$ on the diagonal blocks of $B_{i}, i=1,2$. Therefore

$$
\begin{aligned}
& \mathbb{M}_{n_{1}}(R / \mathcal{P}(R)) \times \cdots \times \mathbb{M}_{n_{t}}(R / \mathcal{P}(R)) \cong \mathbb{M}\left(B_{1}, R\right) / \mathcal{P}\left(\mathbb{M}\left(B_{1}, R\right)\right) \\
& \quad \cong \mathbb{M}\left(B_{2}, R\right) / \mathcal{P}\left(\mathbb{M}\left(B_{2}, R\right)\right) \cong \mathbb{M}_{m_{1}}(R / \mathcal{P}(R)) \times \cdots \times \mathbb{M}_{m_{s}}(R / \mathcal{P}(R)) .
\end{aligned}
$$

By Goldie's theorem $R / \mathcal{P}(R)$ has a (left) quotient ring $Q$ which is semisimple Artinian, and by $(7) \mathbb{M}_{n_{1}}(Q) \times \cdots \times \mathbb{M}_{n_{t}}(Q) \cong \mathbb{M}_{m_{1}}(Q) \times \cdots \times \mathbb{M}_{m_{s}}(Q)$. Writing $Q$ as a finite direct product of full matrix rings over division rings, the desired result follows from the uniqueness of the representation in the Wedderburn-Artin theorem.

The next lemma provides the "bricks" of a structural matrix ring.

Lemma 2.2. If $B$ is a connected Boolean matrix and $R$ an indecomposable ring, then $\mathbb{M}(B, R)$ is an indecomposable ring.

Proof. Since $B$ is connected, it follows readily that $\operatorname{Cen}(\mathbb{M}(B, R))=\operatorname{Cen}(R) \cdot I_{n}$, where $I_{n}$ denotes the $(n \times n)$ identity matrix and $\operatorname{Cen}(R)$ denotes the center of $R$. If $\mathbb{M}(B, R)$ is decomposable, then $I_{n}$ is the sum of two non-zero central idempotents in $\mathbb{M}(B, R)$, and this implies that 1 (the identity of $R$ ) is the sum of two non-zero central idempotents in $R$; a contradiction.

We note that if $\varphi: R_{1} \times \cdots \times R_{n} \rightarrow R_{1}^{\prime} \times \cdots \times R_{m}^{\prime}$ is a ring isomorphism, with $R_{1}, \ldots, R_{n}$ and $R_{1}^{\prime}, \ldots, R_{m}^{\prime}$ indecomposable rings, then $m=n$ since $n$ is the number of central primitive idempotents in $R_{1} \times \cdots \times R_{n}$. Furthermore, induction on $n$ shows that there is a permutation $\sigma \in S_{n}$ such that $\varphi\left(R_{i}\right)=R_{\sigma(i)}^{\prime}, i=1, \ldots, n$.

Next we establish the desired result for the "bricks" of equal order, even over possibly different underlying rings. However, since Lemma 2.3 holds even if $B_{1}$ and $B_{2}$ are not connected, we formulate it as such.

Lemma 2.3. Let $B_{1}$ and $B_{2}$ be Boolean matrices of order $n$, and let $R_{1}$ and $R_{2}$ be indecomposable semiprime Noetherian rings such that $\mathbb{M}\left(B_{1}, R_{1}\right) \cong \mathbb{M}\left(B_{2}, R_{2}\right)$. Then the graphs $\Gamma\left(B_{1}\right)$ and $\Gamma\left(B_{2}\right)$ are isomorphic.

Proof. The discussion preceding Example 1.1 shows that we may assume that $B_{1}$ and $B_{2}$ are blocked triangular, and that it suffices to obtain a permutation $\sigma \in S_{n}$ such that $B_{2}=\sigma\left(B_{1}\right)$. Let $\varphi: \mathbb{M}\left(B_{1}, R_{1}\right) \rightarrow \mathbb{M}\left(B_{2}, R_{2}\right)$ be a ring isomorphism, and let $n_{1}, \ldots, n_{t}$ and $m_{1}, \ldots, m_{s}$ be the orders of the diagonal blocks of $B_{1}$ and $B_{2}$ respectively. We obtain, as in Lemma 2.1 , the induced isomorphism $\bar{\varphi}: \mathbb{M}\left(B_{1}, R_{1}\right) / \mathcal{P}\left(\mathbb{M}\left(B_{1}, R_{1}\right)\right) \rightarrow \mathbb{M}\left(B_{2}, R_{2}\right) / \mathcal{P}\left(\mathbb{M}\left(B_{2}, R_{2}\right)\right)$, and we may consider $\bar{\varphi}$, without loss of generality, as an isomorphism from $\mathbb{M}_{n_{1}}\left(R_{1}\right) \times \cdots \times \mathbb{M}_{n_{t}}\left(R_{1}\right)$ to $\mathbb{M}_{m_{1}}\left(R_{2}\right) \times \cdots \times \mathbb{M}_{m_{s}}\left(R_{2}\right)$. Hence the uniform dimensions of these two direct products of rings are equal, i.e. $\left(n_{1}+\cdots+n_{t}\right) \cdot \mathrm{u} \operatorname{dim} R_{1}=\left(m_{1}+\cdots+m_{s}\right) \cdot \mathrm{u} \operatorname{dim} R_{2}$. By hypothesis $B_{1}$ and $B_{2}$ have the same order, and so u $\operatorname{dim} R_{1}=\mathrm{u} \operatorname{dim} R_{2}$. Since $R_{1}$ and $R_{2}$ are indecomposable, every full matrix ring over $R_{1}$ or over $R_{2}$ is indecomposable. The paragraph following the proof of Lemma 2.2 now shows that $t=s$ and that there is a permutation $\theta \in S_{t}$ such that

$$
\bar{\varphi}\left(\mathbb{M}_{n_{i}}\left(R_{1}\right)\right)=\mathbb{M}_{m_{\theta(i)}}\left(R_{2}\right),
$$

$i=1, \ldots, t$. Looking again at the uniform dimensions $\left(\right.$ of $\mathbb{M}_{n_{i}}\left(R_{1}\right)$ and $\left.\mathbb{M}_{m_{\theta(i)}}\left(R_{2}\right)\right)$, we have $n_{i} \cdot u \operatorname{dim} R_{1}=m_{\theta(i)} \cdot u \operatorname{dim} R_{2}$, and so $n_{i}=m_{\theta(i)}$. 
Let $\sigma \in S_{n}$ be the permutation induced by $\theta$, i.e. the elements involved in the $i$ th diagonal block of $B_{1}$ (viz. $n_{1}+\cdots+n_{i-1}+1, n_{1}+\cdots+n_{i-1}+2, \ldots, n_{1}+\cdots+n_{i-1}+n_{i}$ ) are mapped to the elements involved in the $\theta(i)$ th diagonal block of $B_{2}$ (preserving the order of the elements). Denote by $e_{i}$ (respectively $f_{i}$ ), $i=1, \ldots, t$, the idempotents in $\mathbb{M}\left(B_{1}, R_{1}\right)$ (respectively $\mathbb{M}\left(B_{2}, R_{2}\right)$ ) associated with the diagonal blocks of $B_{1}$ (respectively $B_{2}$ ), i.e. $e_{i}=E_{n_{1}+\cdots+n_{i-1+1}}+E_{n_{1}+\cdots+n_{i-1}+2}+\cdots+E_{n_{1}+\cdots+n_{i-1}+n_{i}}$, $i=1, \ldots, t$, where $E_{k}$ denotes the standard matrix unit (with 1 in position $(k, k)$ and 0 elsewhere). Let $\overline{e_{i}}$ and $\overline{f_{i}}$ denote the images of the $e_{i}$ 's and the $f_{i}$ 's in the factor rings $\mathbb{M}\left(B_{1}, R_{1}\right) / \mathcal{P}\left(\mathbb{M}\left(B_{1}, R_{1}\right)\right)$ and $\mathbb{M}\left(B_{2}, R_{2}\right) / \mathcal{P}\left(\mathbb{M}\left(B_{2}, R_{2}\right)\right)$ respectively. By (8) $\bar{\varphi}\left(\overline{e_{i}}\right)=\overline{f_{\theta(i)}}$, and so $\varphi\left(e_{i}\right)=f_{\theta(i)}+a_{i}$ for some $a_{i} \in \mathcal{P}\left(\mathbb{M}\left(B_{2}, R_{2}\right)\right)$. Since $R_{2}$ is Noetherian, it is clear that $\mathbb{M}\left(B_{2}, R_{2}\right)$ is Noetherian, and so $\mathcal{P}\left(\mathbb{M}\left(B_{2}, R_{2}\right)\right)$ is nilpotent, say $a_{i}^{h}=0$. Consequently $\varphi\left(e_{i}\right)=\left(f_{\theta(i)}+a_{i}\right)^{h} \in \mathbb{M}\left(B_{2}, R_{2}\right) f_{\theta(i)} \mathbb{M}\left(B_{2}, R_{2}\right)$.

Now let $k, l \in\{l, \ldots, n\}$ be such that $B_{1}$ has 1 in position $(k, l)$, and suppose the positions $(k, k)$ and $(l, l)$ are respectively in the $i$ th and $j$ th diagonal blocks of $B_{1}$. Then $e_{i} \mathbb{M}\left(B_{1}, R_{1}\right) e_{j} \neq\{0\}$, which implies that

$$
\begin{aligned}
\{0\} & \neq \varphi\left(e_{i} \mathbb{M}\left(B_{1}, R_{1}\right) e_{j}\right)=\varphi\left(e_{i}\right) \varphi\left(\mathbb{M}\left(B_{1}, R_{1}\right)\right) \varphi\left(e_{j}\right) \\
& \subseteq \mathbb{M}\left(B_{2}, R_{2}\right) f_{\theta(i)} \mathbb{M}\left(B_{2}, R_{2}\right) f_{\theta(j)} \mathbb{M}\left(B_{2}, R_{2}\right) .
\end{aligned}
$$

Therefore $f_{\theta(i)} \mathbb{M}\left(B_{2}, R_{2}\right) f_{\theta(j)} \neq\{0\}$, which means that $B_{2}$ has 1 's in the positions of its $(\theta(i), \theta(j))$ th block, in particular in position $(\sigma(k), \sigma(l))$.

Repeating the above argument with $\varphi^{-1}$ we conclude that $B_{2}=\sigma\left(B_{1}\right)$.

The foregoing results lead to the main result of the sequel:

Theorem 2.4. Let $R$ be a semiprime Noetherian ring, and let $B_{1}$ and $B_{2}$ be Boolean matrices such that $\mathbb{M}\left(B_{1}, R\right)$ and $\mathbb{M}\left(B_{2}, R\right)$ are isomorphic rings. Then the graphs $\Gamma\left(B_{1}\right)$ and $\Gamma\left(B_{2}\right)$ are isomorphic.

Proof. We assume again that $B_{1}$ and $B_{2}$ are blocked triangular. By Lemma $2.1 B_{1}$ and $B_{2}$ have the same order $n$ (say). It suffices to show that there is a permutation $\sigma \in S_{n}$ such that $B_{2}=\sigma\left(B_{1}\right)$. Let $B_{1}^{\prime}, \ldots, B_{p}^{\prime}$ and $B_{1}^{*}, \ldots, B_{q}^{*}$ be the connected components of $B_{1}$ and $B_{2}$ respectively. We may write $R$ as a direct product $A_{1} \times$ $\cdots \times A_{t}$ of indecomposable semiprime Noetherian rings. Let $\varphi: \mathbb{M}\left(B_{1}, R\right) \rightarrow$ $\mathbb{M}\left(B_{2}, R\right)$ be an isomorphism. Then we may regard $\varphi$ as an isomorphism from

$$
\prod_{i=1}^{p}\left(\prod_{j=1}^{t} \mathbb{M}\left(B_{i}^{\prime}, A_{j}\right)\right) \quad \text { to } \prod_{i=1}^{q}\left(\prod_{j=1}^{t} \mathbb{M}\left(B_{i}^{*}, A_{j}\right)\right) \text {. }
$$

By Lemma 2.2 all the factors in (9) are indecomposable, and so the paragraph following the proof of Lemma 2.2 shows that $p=q$.

Now we proceed by induction on $p$. If $p=1$, then $B_{1}=B_{1}^{\prime}, B_{2}=B_{1}^{*}$, and we have $\mathbb{M}\left(B_{1}, A_{1}\right) \cong \mathbb{M}\left(B_{2}, A_{j}\right)$ for some $j$, and so it suffices to invoke Lemma 2.3 according to which there is a permutation $\sigma \in S_{n}$ such that $B_{2}=\sigma\left(B_{1}\right)$. Next, let $p>1$, and assume that the desired result holds if the number of diagonal blocks is $\leq p$. We may assume, without loss of generality, that $\mathrm{u} \operatorname{dim} A_{1}=\max \left(\left\{\mathrm{u} \operatorname{dim} A_{j}: 1 \leq j \leq t\right\}\right)$ and that $\operatorname{order}\left(B_{1}^{\prime}\right)=\max \left(\left\{\operatorname{order}\left(B_{i}^{\prime}\right): 1 \leq i \leq p\right\} \cup\left\{\operatorname{order}\left(B_{i}^{*}\right): 1 \leq i \leq p\right\}\right)$. As before we have $\mathbb{M}\left(B_{1}^{\prime}, A_{1}\right) \cong \mathbb{M}\left(B_{h}^{*}, A_{k}\right)$ for some $h$ and some $k$, and factorising by the prime radicals and considering the uniform dimensions of these two structural matrix rings, we conclude that $B_{1}^{\prime}$ and $B_{h}^{*}$ have the same order (and that u $\operatorname{dim} A_{1}=$ $\mathrm{u} \operatorname{dim} A_{k}$ ). By Lemma 2.3 there is a bijection $\theta$ between the set of elements involved 
in $B_{1}^{\prime}$ (the first diagonal block of $B_{1}$ ) and the set of elements involved in $B_{h}^{*}$ (the $h$ th diagonal block of $\left.B_{2}\right)$. Hence $\mathbb{M}\left(B_{1}^{\prime}, A_{j}\right) \cong \mathbb{M}\left(B_{h}^{*}, A_{j}\right)$ for every $j, j=1, \ldots, t$. Consequently, by (9)

$$
\prod_{i=2}^{p}\left(\prod_{j=1}^{t} \mathbb{M}\left(B_{i}^{\prime}, A_{j}\right)\right) \cong \prod_{\substack{i=1 \\ i \neq h)}}^{p}\left(\prod_{j=1}^{t} \mathbb{M}\left(B_{i}^{*}, A_{j}\right)\right),
$$

and so the induction hypothesis gets us home.

Corollary 2.5. Let $R$ be a commutative Noetherian ring, and let $B_{1}$ and $B_{2}$ be Boolean matrices such that $\mathbb{M}\left(B_{1}, R\right)$ and $\mathbb{M}\left(B_{2}, R\right)$ are isomorphic rings. Then the graphs $\Gamma\left(B_{1}\right)$ and $\Gamma\left(B_{2}\right)$ are isomorphic.

Proof. As in the previous proof we let $B_{1}^{\prime}, \ldots, B_{p}^{\prime}$ denote the connected components of $B_{1}$, and $I_{1}, \ldots, I_{p}$ denote the diagonal identities associated with $B_{1}^{\prime}, \ldots, B_{p}^{\prime}$, i.e. $I_{1}+\cdots+I_{p}$ is the identity of $\mathbb{M}\left(B_{1}, R\right)$. Then $\operatorname{Cen}\left(\mathbb{M}\left(B_{1}, R\right)\right)=R \cdot I_{1} \times \cdots \times R \cdot I_{p}$, and $\mathcal{P}\left(\operatorname{Cen}\left(\mathbb{M}\left(B_{1}, R\right)\right)\right)=\mathcal{P}(R) \cdot I_{1} \times \cdots \times \mathcal{P}(R) \cdot I_{p}$. The two-sided ideal $K_{1}$ of $\mathbb{M}\left(B_{1}, R\right)$ generated by $\mathcal{P}(R) \cdot I_{1} \times \cdots \times \mathcal{P}(R) \cdot I_{p}$ is just $\mathbb{M}\left(B_{1}, \mathcal{P}(R)\right)$, i.e. the set of matrices in $\mathbb{M}\left(B_{1}, R\right)$ with entries from $\mathcal{P}(R)$. If $\varphi: \mathbb{M}\left(B_{1}, R\right) \rightarrow \mathbb{M}\left(B_{2}, R\right)$ denotes an isomorphism, then $\varphi\left(K_{1}\right)=K_{2}$, with $K_{2}$ the two-sided ideal of $\mathbb{M}\left(B_{2}, R\right)$ generated by $\mathcal{P}\left(\operatorname{Cen}\left(\mathbb{M}\left(B_{2}, R\right)\right)\right)$. Hence

$$
\mathbb{M}\left(B_{1}, R / \mathcal{P}(R)\right) \cong \mathbb{M}\left(B_{1}, R\right) / K_{1} \cong \mathbb{M}\left(B_{2}, R\right) / K_{2} \cong \mathbb{M}\left(B_{2}, R / \mathcal{P}(R)\right),
$$

and so the result follows from Theorem 2.4.

If we drop the condition of being Noetherian in Corollary 2.5, then even if we require equal orders for $B_{1}$ and $B_{2}$, the graphs $\Gamma\left(B_{1}\right)$ and $\Gamma\left(B_{2}\right)$ need not be isomorphic. For example, let $R$ be commutative such that $R \cong R \times R$. Then $\mathbb{M}_{2}(R) \times R \times R \times R \cong \mathbb{M}_{2}(R) \times \mathbb{M}_{2}(R) \times R$.

Theorem 2.4 also provides a uniqueness result for the representation of a left Artinian CI-prime ring (see the definition of a complete blocked triangular Boolean matrix in Section 1 and the corresponding definition of a complete blocked triangular matrix ring in [5]).

Corollary 2.6. Let $D_{1}$ and $D_{2}$ be division rings such that the complete blocked triangular matrix rings $\mathbb{M}\left(B_{1}, D_{1}\right)$ and $\mathbb{M}\left(B_{2}, D_{2}\right)$ are isomorphic. Then $B_{1}=B_{2}$ and $D_{1} \cong D_{2}$.

Proof. Factorising by the prime radicals, the uniqueness of the representation in the Wedderburn-Artin theorem ensures that $D_{1} \cong D_{2}$. Now apply Theorem 2.4, and note that if the graphs $\Gamma\left(B_{1}\right)$ and $\Gamma\left(B_{2}\right)$ are isomorphic, with $B_{1}$ and $B_{2}$ complete blocked triangular Boolean matrices, then $B_{1}=B_{2}$.

We conclude by mentioning that we are of the opinion that the condition of being semiprime can be dropped in Theorem 2.4, but we have neither been able to come up with a proof, nor have we been able to find a counterexample.

\section{ACKNowledgments}

The first author gratefully acknowledges helpful discussions with J. C. Robson regarding Example 1.1. The authors express their gratitude towards the Foundation for Research Development of South Africa which made the first author's visit to the University of Stellenbosch possible. 


\section{REFERENCES}

1. A. W. Chatters, Non-isomorphic rings with isomorphic matrix rings, Proc. Edinburgh Math. Soc. 36 (1993), 339-348. MR 94b:16035

2. S. P. Coelho, Automorphism groups of certain structural matrix rings, Comm. Algebra 22 (1994), 5567-5586. CMP 95:02.

3. S. Jøndrup, The group of automorphisms of certain subalgebras of matrix algebras, J. Algebra 141 (1991), 106-114. MR 92h:16034

4. L. S. Levy, J. C. Robson, and J. T. Stafford, Hidden matrices, Proc. London Math. Soc. 69 (1994), 277-308. MR 95d:16026

5. M. S. Li and J. M. Zelmanowitz, Artinian rings with restricted primeness conditions, J. Algebra 124 (1989), 139-148. MR 90g:16006

6. J. C. McConnell and J. C. Robson, Noncommutative Noetherian rings, Wiley, New York, 1967.

7. L. van Wyk, Special radicals in structural matrix rings, Comm. Algebra 16 (1988), 421-435. MR 89a:16009

Facultatea de Matematica, University of Bucharest, Str. Academiei 14, R 70109, Bucharest 1, Romania

Department of Mathematics, University of Stellenbosch, Stellenbosch 7600, South Africa

Current address, L. van Wyk: Department of Mathematics, Texas A \& M University, College Station, Texas 77843-3368

E-mail address: lvw@maties.sun.ac.za 